ERRATA DO VOLUME 10 (3)

\begin{tabular}{ccll}
\hline Página & Linha & Onde se lê & Leia-se \\
\hline 429 & 18 e 32 & $\mathrm{~L}$ & $\mathrm{~L}_{\infty}$ \\
431 & 27 & $\mathrm{~L}$ & $\mathrm{~L}_{\infty}$ \\
434 & Legenda & $\mathrm{L}$ & $\mathrm{L}_{\infty}$ \\
435 & 35 & M. amazonicum & M. amazonicum (Heller, 1862) \\
435 & 35 & (Wiegmon, 1836) & (Wiegmann, 1836) \\
435 & 36 & Linnaeus, 1758 & (Linnaeus, 1758) \\
\hline
\end{tabular}

\title{
ERRATA DO VOLUME 10 (4)
}

\begin{tabular}{|c|c|c|c|}
\hline Página & Linha & Onde se lê & Leia-se \\
\hline 595 & 06 & \multirow{5}{*}{ Prhytocoris } & \multirow{5}{*}{ Phytocoris } \\
\hline 600 & 37 & & \\
\hline 601 & 22 & & \\
\hline 602 & 11 & & \\
\hline 603 & 01 & & \\
\hline 597 & 04 & \multirow{5}{*}{ Phitocoris } & \multirow{5}{*}{ Phvtocoris } \\
\hline 600 & 35 & & \\
\hline 601 & 21 & & \\
\hline 602 & 10 e 41 & & \\
\hline 603 & 29 & & \\
\hline 619 & 12 & $0,50 \mu \mathrm{m}$ & $0,59 \mu \mathrm{m}$ \\
\hline 619 & 26 & IVAN HORST & (VAN DER HORST \\
\hline 620 & 10 & (SORVAL MR-1) & (SORVAL MT-1) \\
\hline 620 & 17 & (Figs $1 a, b ; 2 a, d)$ & (Figs $1 \mathrm{a}, \mathrm{b} ; 2 \mathrm{a}, \mathrm{b}$ ) \\
\hline 620 & 39 & (Fig. 2s) & (Fig. 2a) \\
\hline 621 & 11 & on A-tubules & in A-tubules \\
\hline 622 & 09 e 18 & M. planatus & M. platanus \\
\hline 622 & 10 e 19 & (EIRA-STOFELLA & (EIRAS-STOFELLA \\
\hline 622 & 21 & (1978) & $(1968)$ \\
\hline 622 & 22 & head rounded & head is normally rounded \\
\hline 623 & 14 & m. curema & M. curema \\
\hline 625 & 02 & types different & types of different \\
\hline 626 & 13 & and M.liza & and in M.liza \\
\hline 626 & 17 & M. aurata & L. aurata \\
\hline 626 & 19 & (EIRAS-STOFELLS & (EIRAS-STOFELLA \\
\hline 626 & 21 & $\begin{array}{l}\text { contains } A \text { - and } B \text { - tubules } \\
\text { being more electron-dense } \\
\text { than } B \text { - tubules }\end{array}$ & $\begin{array}{l}\text { contains } A \text { and } B \text { - tubules, the } \\
\text { A-tubules being more } \\
\text { electron-dense than the } B \text { - } \\
\text { tubules }\end{array}$ \\
\hline 626 & 27 & ara & are \\
\hline 627 & $\mathrm{O} 2$ & 9181 & 1981 \\
\hline Página & Figura & Onde se lê & Leia-se \\
\hline 622 & 2 & (h) head & (H) head \\
\hline 622 & 2 & (h) hilus. & (h) nuclear hilus. \\
\hline 623 & $3-4$ & flagellum; & flagellum (f): \\
\hline 625 & $6-7$ & $(\rightarrow)$ microtubules & $\left({ }^{* *}\right)$ microtubules \\
\hline
\end{tabular}

ERRATA DO VOLUME 11 (1)

\begin{tabular}{llll}
\hline Página Linha & Onde se lê & Leia-se \\
\hline 02 & 20 & $\begin{array}{l}\text { MCZC - Museum of Comparative } \\
\text { Zoology, Cambridge, Massachusetts, } \\
\text { USA }\end{array}$ & $\begin{array}{l}\text { ZMCl - Zoological Museum, } \\
\text { Cambridge, Inglaterra; }\end{array}$ \\
Em todo o texto & MCZC exam. aut. & ZMCl exam. aut. \\
\hline
\end{tabular}

